\title{
Efecto de bloques con propionato de calcio sobre respuestas productivas en corderos y GEI in vitro
}

\author{
Nallely Sánchez $\mathrm{L}^{1} \bowtie(\mathbb{0})$ M.Sc; Germán Mendoza $\mathrm{M}^{1} \otimes(\mathbb{0})$ Ph.D; José Martínez $\mathrm{G}^{2} \bowtie(\mathbb{0}$ Ph.D; \\ Pedro Hernández $\mathrm{G}^{3} \otimes \mathbb{0}$ Ph.D; Luis Miranda R ${ }^{4}$ Ph.D; Oscar Villarreal EB ${ }^{5 *} \otimes(\mathbb{0}$ Ph.D.
}

\author{
${ }^{1}$ Universidad Autónoma Metropolitana Xochimilco, Departamento de Producción Agrícola y Animal, Doctorado en Ciencias Agropecuarias, \\ CDMX., 04960, México. \\ 2Universidad Autónoma Metropolitana Xochimilco, Departamento de Producción Agrícola y Animal, CDMX, México, 04960. \\ 3Universidad del Estado de México, Centro Universitario Amecameca, 56900, México. \\ 4Universidad Autónoma Chapingo, Departamento de Zootecnia, México, Edo. Mex., 56230, México. \\ ${ }^{5}$ Benemérita Universidad Autónoma de Puebla, Facultad de Medicina Veterinaria y Zootecnia. Km. 7.5 Carretera Tecamachalco, Cañada \\ Morelos, El Salado Tecamachalco, Puebla, México 75480.
}

*Correspondence: dr.oscarvillarreal@gmail.com

\section{RESUMEN}

Objetivo. Evaluar bloques multinutricionales con y sin propionato de calcio ( $\mathrm{Pr}$-Ca) en la digestibilidad y cambios de peso de corderos y en la emisión de gases in vitro. Materiales y métodos. Se utilizaron doce borregos (20.17 \pm 2.35 Katahdin $x$ criollo) en tres tratamientos: Dieta basal (DB $70 \%$ paja de avena; $30 \%$ concentrado), DB+ Bloque sin Pr$\mathrm{Ca}$ y $\mathrm{DB}+$ Bloque con $1.5 \%$ de Pr-Ca, por 50 días. Se midió producción de gas (PG) in vitro y parámetros de cinética (Vmax, S, Lag). Se estimó la digestibilidad, $\mathrm{CH}_{4}$ y $\mathrm{CO}_{2}$ in vitro e in vivo. Resultados. El consumo de materia seca fue menor $(p<0.0001)$ en borregos sin bloque $(753 \mathrm{~g} / \mathrm{d})$ en comparación con bloque sin $(839 \mathrm{~g})$ o con Pr-Ca (828 g) al incluir bloques aumentó metano $(16.16$ y $16.18 \mathrm{~g} / \mathrm{d} ; 0$ y $1.5 \% \mathrm{Pr}$-Ca respectivamente) que con DB (13.93 g/d). La PG in vitro fue mayor $(p=0.0001)$ con la DB $(380.76 \mathrm{ml})$ sin diferencias entre bloques $(335.76$ y $341.13 \mathrm{ml}, 0$ y $1.5 \%$ Pr-Ca respectivamente), y la DB tuvo mayor $(\mathrm{p}=0.0001)$ producción de $\mathrm{CH}_{4}(47.16 \mathrm{~mol})$ y $\mathrm{CO}_{2}$ (200.04 mol) que con bloques (42.25 y 41.58 mol CH${ }_{4} ; 179.21$ y 176.39 moles $\mathrm{CO}_{2} ; 0$ y $1.5 \% \mathrm{Ca}-\mathrm{Pr}$ respectivamente). Conclusiones. La suplementación con bloques mejora el consumo de materia seca. Los bloques disminuyeron la producción de gas in vitro e incrementaron la digestibilidad reduciendo $\mathrm{CH}_{4}$ y $\mathrm{CO}_{2}$.

Palabras clave: Aditivo, gas efecto invernadero, rumiante, suplemento. (Fuente:CAB)

\begin{abstract}
Objective. Evaluate multinutritional blocks with and without calcium propionate (Pr-Ca) in digestibility and live weight changes of lambs and in the green house emission in vitro. Materials and methods. Twelve sheep were used $(20.17 \pm 2.35$ Katahdin $x$ criollo) in three treatments: Basal diet (BD 70\% oat straw, 30\% concentrate), BD+ Block without $\mathrm{Ca}-\mathrm{Pr}$ and $\mathrm{BD}+\mathrm{Block}$ with $1.5 \% \mathrm{Ca}-\mathrm{Pr}$, it was evaluated for 50 days. In vitro gas production (GP) and kinetic parameters were estimated (Vmax, $\mathrm{S}, \mathrm{Lag}$ ) according to consumption. Digestibility, $\mathrm{CH}_{4}$ and $\mathrm{CO}_{2}$ in vitro and in vivo were estimated. Results. The dry matter intake was the lowest $(p<0.0001)$ in lambs without block $(753 \mathrm{~g} / \mathrm{d}) \mathrm{compared}$ lambs supplemented with block without $(839 \mathrm{~g})$ or with Ca-Pr $(828 \mathrm{~g})$ by including blocks increased methane (16.16 and $16.18 \mathrm{~g} / \mathrm{d} ; 0$ and $1.5 \%$ Ca-Pr respectively) than with BD $(13.93 \mathrm{~g} / \mathrm{d})$. The GP in vitro was higher ( $\mathrm{p}=0.0001)$ with the BD $(380.76, \mathrm{ml})$ than with blocks without differences among blocks (335.76 and $341.13 \mathrm{ml}, 0$ and $1.5 \%$ $\mathrm{Ca}-\mathrm{Pr}$ respectively), and the $\mathrm{BD}$ had higher $(\mathrm{p}=0.0001)$ production of $\mathrm{CH}_{4}(47.16 \mathrm{~mol})$ and $\mathrm{CO}_{2}(200.04 \mathrm{~mol})$ than with blocks (42.25 and $41.58 \mathrm{~mol} \mathrm{CH}_{4} ; 179.21$ and $176.39 \mathrm{~mol} \mathrm{CO}_{2} ; 0$ and $1.5 \% \mathrm{Ca}$-Pr respectively). Conclusions. Block supplementation improved dry matter intake. Blocks reduced in vitro gas production and increased digestibility by reducing $\mathrm{CH}_{4}$ and $\mathrm{CO}_{2}$.
\end{abstract}

Keywords: additive, greenhouse gas, ruminant, supplement. (Source:CAB)

Como citar (Vancouver).

Sánchez LN, Mendoza MG, Martínez GJ, Hernández GP, Miranda RL, Villarreal EBO. Efecto de bloques con propionato de calcio sobre respuestas productivas en corderos y GEI in vitro. Rev MVZ Cordoba. 2019; 24(2):7188-7192. DOI: https://doi.org/10.21897/rmvz.1229

(C)EI (los) autor (es), Revista MVZ Córdoba 2019. Este artículo se distribuye bajo los términos de la licencia internacional Creative Commons Attribution 4.0 (https://creativecommons.org/licenses/by-sa/4.0/), que permite el uso sin restricciones, la distribución y la reproducción en cualquier medio, siempre que se otorgue el crédito apropiado al autor o autores originales y la fuente. 


\section{INTRODUCCIÓN}

Se han realizado estudios para evaluar la contribución de los rumiantes a las emisiones de gases con efecto invernadero (GEI) y las alternativas para mitigar el problema (1), los cuales representan arriba del $12 \%$ de la energía consumida (2). Para reducir estas pérdidas y hacer que la producción sea más eficiente se ha evaluado la utilización de aditivos $(3,4)$ los cuales en ocasiones no mejoran el rendimiento (5) o no impactan en la fermentación (6) y los más eficientes como los ionóforos han sido prohibidos porque son antibióticos.

El propionato de calcio (Pr-Ca) como una fuente de alimentación no convencional ha sido utilizado en corderos para reducir el uso de granos, incrementando el propionato ruminal (7). Su potencial para reducir el metano se explica porque durante su disociación captura el ion de hidrógeno reduciendo su disponibilidad para formar metano (8). Las emisiones de GEI pueden reducirse si se mejora la digestibilidad, lo cual se alcanza cubriendo las necesidades nutricionales, particularmente en rumiantes alimentados con dietas con forrajes de baja calidad (9). Una opción para cubrir limitantes en la alimentación es suplementando con bloques multinutricionales (BM) que han sido evaluados en todo el mundo (10), sin embargo, la respuesta no ha sido constante porque durante muchos años la FAO promovió una fórmula de bloque para todas las condiciones (11) cuando los requerimientos de nutrientes son diferentes por cada etapa fisiológica y con diversidad de dietas basales. Por lo tanto, el objetivo de este experimento fue evaluar bloques multinutricionales formulados con el fin de mejorar el crecimiento de los corderos alimentados con una dieta basal de bajo valor nutritivo, con o sin $\mathrm{Pr}-$ $\mathrm{Ca}$, evaluando el impacto en el crecimiento del cordero, digestibilidad y en las emisiones de GEI in vivo e in vitro.

\section{MATERIALES Y MÉTODOS}

Ubicación. Este trabajo se realizó en las instalaciones del Centro Universitario UAEM en la Posta Zootécnica de la Universidad Autónoma del Estado de México, Amecameca, Estado de México ubicada en la zona sur del oriente del Estado de México.

Condiciones climáticas. Clima templado subhúmedo con temperatura media anual de $14.7^{\circ} \mathrm{C}$.

Animales. Se usaron doce corderos Katahdin $x$ criollo (20.17 \pm 2.35 peso inicial) alojados en jaulas individuales con acceso al alimento y agua limpia ad libitum. Este trabajo se realizó bajo lineamientos del Comité Académico del Departamento de Ciencia Animal, de acuerdo con las regulaciones establecidas por la Ley de Protección Animal del Estado de México, México.

Tratamientos. El experimento duró 50 días, los corderos fueron distribuidos en un Diseño Completamente al Azar ( $n=4$ corderos) en tres tratamientos: dieta basal sin suplementar (DB: $70 \%$ rastrojo de maíz, $30 \%$ concentrado, Tabla 1 ), DB con acceso a bloque multinutricional con o sin $1.5 \%$ de Pr-Ca (Alimentaria Mexicana Bekarem, Ciudad de México) (Tabla 2).

El alimento y los bloques fueron ofrecidos ad libitum. La dieta basal fue diseñada para simular a las que se ofrecen
Tabla 1. Composición de la dieta basal (materia seca) forraje concentrado (70:30)

\begin{tabular}{cc}
\hline Ingredientes & Inclusión, \% \\
\hline Rastrojo de maíz & 70.00 \\
Grano de maíz & 9.22 \\
Pasta de soya & 11.00 \\
Grano de Sorgo & 8.80 \\
Sal & 1.00 \\
\hline Total & 100.00 \\
\hline
\end{tabular}

Tabla 2. Formulación de bloques multinutricionales.

\begin{tabular}{ccc}
\hline Melaza & $\begin{array}{c}\text { Propionato de } \\
\text { calcio 0\% }\end{array}$ & $\begin{array}{c}\text { Propionato de } \\
\text { calcio 1.5\% }\end{array}$ \\
\hline Urea & 31.81 & 31.91 \\
Propionato de calcio & 9.09 & 9.11 \\
Pasta de soya & 4.54 & 1.50 \\
Harina de canola & 2.27 & 4.55 \\
Harina de pescado & 2.27 & 2.27 \\
Sal & 4.54 & 2.27 \\
Premezcla minerala & 4.54 & 4.55 \\
Cal & 4.54 & 4.55 \\
Cemento & 4.54 & 4.55 \\
Premezcla mineral orgánicab & 0.90 & 4.55 \\
Salvado de trigo & 9.09 & 0.91 \\
Cascarilla de soya & 4.54 & 9.11 \\
Grano de maíz & 8.18 & 4.55 \\
Grano de trigo & 9.09 & 6.38 \\
\hline
\end{tabular}

a Vitasal Engorda Ovinos Plus contenía: Ca 270 g, P 30 g, Mg 7.5 g, Na $65.6 \mathrm{~g}, \mathrm{Cl} 100 \mathrm{~g}, \mathrm{~K} 0.5 \mathrm{~g}, \mathrm{~S} 42 \mathrm{mg}$, Fe $978 \mathrm{mg}, \mathrm{Zn} 3000 \mathrm{mg}$, Se $20 \mathrm{mg}$, Co $15 \mathrm{mg}$, vitamina A 35000 UI, vitamina D 1500000 IU y vitamina E 150 UI. b Ovy ways 3 contenía: Selenio $590 \mathrm{mg}$, Cromo $990 \mathrm{mg}$, Cobre $1500 \mathrm{mg}$, Hierro 3000 mg, Zinc 3000 mg, Manganeso 3000 mg, Células de levadura vivas.

en unidades de producción de tipo familiar (11\% proteína; $2.7 \mathrm{Mcal} / \mathrm{kg}$ MS EM). Los bloques multinutricionales (BM) fueron formulados para que al suplementarse cumplieran los requerimientos nutricionales para corderos de acuerdo con el NRC (12) para una ganancia de peso de $150 \mathrm{~g} / \mathrm{d}$ estimando un consumo de $100 \mathrm{~g} / \mathrm{d}$ bloque. La dieta basal y bloques fueron analizados para determinar: materia seca (MS), materia orgánica (MO), proteína cruda (PC) (13), fibra detergente neutra (FDN) y fibra detergente ácida (FDA) (14) (Tabla 3).

Tabla 3. Composición química de la dieta basal y bloques multinutricionales.

\begin{tabular}{cccc} 
& & \multicolumn{2}{c}{ Bloques multinutricionales } \\
MS, \% & 89.28 & 89.32 & 89.26 \\
Cenizas, \% & 4.47 & 7.63 & 7.73 \\
MO, \% & 95.53 & 92.36 & 92.25 \\
PC, \% & 11.05 & 11.53 & 11.76 \\
FDN, \% & 40.28 & 36.71 & 36.50 \\
FDA, \% & 14.85 & 13.77 & 13.57 \\
EE, \% & 3.13 & 2.72 & 2.72
\end{tabular}

Pr-Ca: Propionato de calcio en bloque; MS: materia seca; MO: materia orgánica; PC: proteína cruda; FDN: fibra detergente neutra; FDA: fibra detergente ácida; $\mathrm{EE}$ : extracto etéreo 
Los corderos fueron pesados con 12 horas de ayuno. En el día 24 las muestras fecales fueron recolectadas por cuatro días consecutivos para determinar la digestibilidad del tracto total de la MS usando ceniza de ácido insoluble como marcador interno (15).

Estimación $\mathbf{C H}_{4}$ y $\mathbf{C O}_{2}$ in vivo. Las ecuaciones de IPCC fueron usadas (16) para estimar el $\mathrm{CH}_{4}$ ruminal utilizando el factor de emisión anual (FE) por cordero, donde el Ym o fracción de la energía bruta del alimento transformado a $\mathrm{CH}_{4}$ fue calculado utilizando la digestibilidad de cada tratamiento con ecuaciones para cordero (17). Las emisiones de dióxido de carbono fueron estimadas del consumo de carbohidratos digestibles (18), que se usaron para estimar los moles de hexosa fermentados en el rumen usando el peso molecular de glucosa anhidra (19). El patrón de fermentación fue de la relación forraje: concentrado y por las ecuaciones estequiométricas de Wolin (20) fueron calculados los moles de $\mathrm{CO}_{2}$ producidos.

Cinética de gas in vitro. La producción de gas derivada de la fermentación ruminal fue determinada por la técnica de gas in vitro (21). La DB se utilizó como sustrato incubado con cada BM en una proporción del $5 \%$ de la dieta basal. Antes de la incubación, los sustratos se secaron a $55^{\circ} \mathrm{C}$ por $48 \mathrm{~h}$ en un horno y molido ( $<2 \mathrm{~mm}$ ). En botellas ámbar de $125 \mathrm{ml}$ de capacidad se colocaron $500 \mathrm{mg}$ de cada tratamiento. Después se incubaron en condiciones anaeróbicas con $90 \mathrm{ml}$ de un inóculo diluido (1:10) de bacterias del rumen obtenidas de dos corderos en ayuno. Los frascos fueron herméticamente sellados e incubados a $39^{\circ} \mathrm{C}$ por $72 \mathrm{~h}$ en baño maría. El volumen de gas producido se registró a las $2,4,6,8,12,16,20$, $24,30,36,42,48,60$ y 72 h y los valores de presión transformados a volumen de gas con la ecuación de regresión lineal, usada para estimar los parámetros de la cinética de producción de gas: volumen máximo de gas (Vm; $\mathrm{mL} \mathrm{g}^{-1} \mathrm{MS}$ del sustrato), tasa de producción de gas $\left(S ; h^{-1}\right)$ y el tiempo lag de la fermentación $(L ; h)$, con el modelo: $\mathrm{Vo}=\mathrm{Vm} /\left(1+\mathrm{e}\left(2-4^{*} \mathrm{~s} *(\mathrm{t}-\mathrm{L})\right)\right)(21)$. Al final de la fermentación se obtuvo la materia seca residual (MS) para calcular la digestibilidad in vitro de la materia seca (DIVMS) a las 72 h de incubación, cada tratamiento incubado por triplicado.

Estimación $\mathbf{C H}_{4}$ y $\mathbf{C O}_{2}$ in vitro. Se estimaron a partir del volumen máximo de gas, los ácidos grasos de cadenas cortas fueron calculados con la ecuación de Getachew (22) y la proporción de $\mathrm{CH}_{4}$ y dióxido de carbono los factores estequiométricos utilizados fueron $0.538 \mathrm{mmol}$ para $\mathrm{CO}_{2}$ y $0.348 \mathrm{mmol} \mathrm{CH}_{4}$ que se han descrito en otros estudios in vitro (23).

Diseño experimental. Los datos de cada experimento se analizaron de acuerdo a un Diseño Completamente al Azar con un modelo lineal generalizado usando cada borrego como unidad experimental en el experimento in vivo o los parámetros del ensayo obtenidos de las incubaciones in vitro, considerando los tratamientos como efectos fijos y los errores aleatorios asociados con cada observación. Las medias de los tratamientos se compararon mediante la prueba de Tukey $(p=0.05)$. Para el experimento in vivo el peso inicial fue analizado como covariable utilizando el software JMP (24). Los parámetros de cinética de gas in vitro fueron estimados para $\mathrm{Vo}=\mathrm{Vm} /\left(1+\mathrm{e}\left(2-4 * \mathrm{~s}^{*}(\mathrm{t}-\mathrm{L})\right)\right)$ usando modelos no lineales del JMP. Se estimó la correlación simple entre los resultados de GEI in vivo e in vitro.

\section{RESULTADOS}

La suplementación con BM incrementó el consumo de materia seca $(p<0.01)$ en $10 \%$, sin embargo, no se encontraron diferencias en otras variables (Tabla 4). La suplementación con bloques no redujo las emisiones diarias de metano y $\mathrm{CO}_{2}$ debido al mayor consumo de alimento. Los corderos que consumieron bloques con $\mathrm{Pr}$-Ca no redujeron el consumo ni tuvieron efecto sobre las emisiones estimadas de GEI.

Tabla 4. Rendimiento productivo emisión de metano y dióxido de carbono de corderos suplementados con bloques con o sin propionato de calcio.

\begin{tabular}{|c|c|c|c|c|c|}
\hline & \multirow[b]{2}{*}{ Control } & \multicolumn{2}{|c|}{$\begin{array}{c}\text { Bloques } \\
\text { Multinutricionales } \\
\end{array}$} & \multirow[b]{2}{*}{ EEM } & \multirow[b]{2}{*}{ P-value } \\
\hline & & Pr-Ca 0\% & Pr-Ca $1.5 \%$ & & \\
\hline Peso inicial, kg & 21.23 & 19.70 & 19.60 & 0.40 & 0.59 \\
\hline Peso final, $\mathrm{kg}$ & 23.98 & 23.25 & 23.52 & 0.74 & 0.94 \\
\hline CMS, g & $753^{c}$ & $839^{b}$ & $828^{a}$ & 0.01 & 0.0001 \\
\hline Consumo bloque, $\mathrm{g}$ & $0.0^{\mathrm{b}}$ & $82^{\mathrm{a}}$ & $86^{a}$ & 6.48 & 0.0001 \\
\hline GDP, g & 0.098 & 0.126 & 0.140 & 0.34 & 0.36 \\
\hline $\mathrm{CA}$ & 9.94 & 6.98 & 6.39 & 0.37 & 0.51 \\
\hline $\begin{array}{l}\text { Digestibilidad } \\
\text { MS, } \%\end{array}$ & 76.87 & 80.04 & 81.22 & 0.28 & 0.32 \\
\hline $\mathrm{CH}_{4}, \mathrm{~g} / \mathrm{d}$ & $13.93^{b}$ & $16.16^{\mathrm{a}}$ & $16.18^{\mathrm{a}}$ & 0.003 & 0.004 \\
\hline $\mathrm{CO}_{2}, \mathrm{~g} / \mathrm{d}$ & $55.29^{b}$ & $64.15^{a}$ & $64.24^{\mathrm{a}}$ & 0.003 & 0.004 \\
\hline
\end{tabular}

Pr-Ca: Propionato de calcio en bloque; EEM: error estándar de la media; CMS: consumo de materia seca; GDP: ganancia diaria de peso; CA: conversión alimenticia, $\mathrm{MS}$ : materia seca; $\mathrm{CH}_{4}$ : metano; $\mathrm{CO}_{2}$ : dióxido de carbono.

ab Medias con diferentes superíndices son diferentes $(p<0.05)$

En la Tabla 5 se muestran los parámetros de gas in vitro. La dieta basal resultó con un alto volumen de gas $(\mathrm{p}<0.001)$ y como consecuencia, se produjeron más moles de $\mathrm{CH}_{4}$ y $\mathrm{CO}_{2}(p<0.0001)$. No hubo diferencias entre los bloques por la inclusión de Pr-Ca. La digestibilidad in vitro no fue afectada por la suplementación con bloque. Los valores de digestibilidad in vitro fueron correlacionados con los observados in vivo $(r=0.996, p=0.054)$, y la digestibilidad in vivo está asociada con la ganancia diaria de peso $(r=0.997, p=0.0434)$. Las emisiones de $\mathrm{CH}_{4} \mathrm{y}$ $\mathrm{CO}_{2}$ fueron positivamente correlacionadas con el consumo de materia seca $(r=0.992, p=0.07 ; r=0.992, p=0.08)$. El $\mathrm{CH}_{4}$ y $\mathrm{CO}_{2}$ in vivo e in vitro mostraron una alta correlación negativa $(r=-0.99, p=0.07 ; r=-0.99, p=0.07)$.

Tabla 5. Parámetros de producción de gas in vitro, metano y dióxido de carbono, de la dieta basal incubada con bloques con o sin propionato de calcio.

\begin{tabular}{|c|c|c|c|c|c|}
\hline & \multirow[b]{2}{*}{ Control } & \multicolumn{2}{|c|}{$\begin{array}{c}\text { Bloques } \\
\text { Multinutricionales } \\
\end{array}$} & \multirow[b]{2}{*}{ EEM } & \multirow[b]{2}{*}{ p-value } \\
\hline & & $\mathrm{Pr}-\mathrm{Ca} \%$ & Pr-Ca .5\% & & \\
\hline Vmax, ml & $380.76^{a}$ & $335.76^{b}$ & $341.13^{b}$ & 0.567 & 0.0044 \\
\hline $\mathrm{S}, \mathrm{h}^{-1}$ & $0.030^{\mathrm{b}}$ & $0.033^{a}$ & $0.032^{\mathrm{a}}$ & 0.108 & 0.0005 \\
\hline Lag, h & 2.87 & 3.10 & 2.79 & 0.059 & 0.1283 \\
\hline DIVMS, \% & 60.96 & 63.89 & 64.58 & 0.622 & 0.0734 \\
\hline $\mathrm{CH}_{4}, \mathrm{~mol}$ & $47.16^{a}$ & $41.58^{b}$ & $42.25^{b}$ & 0.568 & 0.0044 \\
\hline $\mathrm{CO}_{2}, \mathrm{~mol}$ & $200.04^{a}$ & $176.39^{b}$ & $179.21^{b}$ & 0.567 & 0.0044 \\
\hline
\end{tabular}

Ca-Pr: Propionato de calcio en bloque; EEM, error estándar de la media; Vmax: volumen máximo, S: tasa de producción de gas, Lag: tiempo de retraso, DIVMS: digestibilidad in vitro de la materia seca.

ab Medias con diferentes superíndices difieren $(p<0.05)$. 


\section{DISCUSIÓN}

Como se observó en este experimento, se ha reportado que los BM estimulan el consumo (25) pero hay estudios donde no tuvieron efecto (26). La composición de los bloques puede modificar el consumo (10), existiendo interacciones entre contenido de nutrientes en el bloque y en la dieta basal. En esos estudios, cuando mejora el consumo, generalmente se han observado mayores ganancias diarias o peso final y en algunos casos, esto se asocia con una mayor digestibilidad (9) y consumo de nutrientes. En este estudio la ganancia fue mejorada en un $35 \%$ pero el bajo número de repeticiones y la variación no permitieron detectar diferencias. El tipo de bloque puede tener diferentes efectos en el consumo y la digestibilidad. Al modificar la fuente de energía afectó el consumo de bloque sin afectar la digestibilidad (25).

La incorporación de Pr-Ca en el BM no mejoró el rendimiento del cordero ni las emisiones de dióxido de carbono. $\mathrm{Si}$ los valores de $\mathrm{CH}_{4}$ y $\mathrm{CO}_{2}$ in vivo fueran expresados por $\mathrm{kg}$ de MS consumida resultarían valores similares (DB 1.84, 1.95 y 1.92 por BM con 0 ó $1.5 \%$ PrCa) lo que indica que el consumo de bloques y sus aditivos fueron insuficientes para modificar la fermentación ruminal. En otras evaluaciones, el Pr-Ca no afectó el consumo ni el rendimiento del cordero, 1\% MS (7) y arriba de $5.5 \%$ MS (27) la cantidad consumida en el BM estuvo por debajo de esos estudios. Los resultados de gas in vitro indican que los $\mathrm{BM}$ reducirían el $\mathrm{CH}_{4}$, pero los valores in vivo contradicen esto ya que aumentan el consumo. Se debe tener precaución al extrapolar los resultados de los estudios de gas in vitro donde no se presentan datos in vivo (28). Con respecto a otros parámetros de gas in vitro, se ha reportado que el $1 \% \operatorname{Pr}-\mathrm{Ca}$ aumenta la fase Lag pero no afecta el patrón de fermentación o pérdidas de $\mathrm{CH}_{4}$ (29). En otro estudio in vitro con $10 \%$ de Pr-Ca, el volumen de gas aumentó el cual fue atribuido a los efectos sobre $\mathrm{pH}$ y a la presión osmótica (8). En una evaluación in vivo con toros recibieron $20 \mathrm{~g} / \mathrm{d}$ de Pr-Ca, no afectaron la fermentación ni la población microbiana (30), pero la dosis fue muy baja.

El uso de BM podría reducir el tiempo de los corderos para alcanzar el peso final de 44 a 60 días suplementando sin o con $\mathrm{Pr}-\mathrm{Ca}$ respectivamente, reduciendo emisiones diarias, y podría ser una alternativa para reducir las emisiones globales de GEI. La mayoría de los estudios se centran en datos diarios, pero es importante considerar los efectos y su impacto sobre el calentamiento global en términos de tiempo (21).

En conclusión, la suplementación con bloques multinutricionales en dietas de baja calidad mejoró el consumo. In vitro los bloques redujeron la producción de gas y aumentaron la digestibilidad, por lo que potencialmente podrían reducir las emisiones de metano y dióxido de carbono.

\section{Conflicto de intereses}

Los autores no declaramos ningún conflicto de intereses.

\section{Agradecimientos}

Al Consejo Nacional de Ciencia y Tecnología (CONACyT México). Esta investigación fue parcialmente respaldada por PROFIDES SEP México y el Programa para el Desarrollo Profesional Docente, para el Tipo Superior (PRODEP). Los autores agradecen a Grupo Biotecap S.A. de C.V. por la donación de minerales orgánicos.

\section{REFERENCIAS}

1. Patra A, Park T, Kim M, Yu Z. Rumen methanogens and mitigation of methane emission by antimethanogenic compounds and substances. J Anim Sci Biotechnol. 2017; 8(1):1-18. https://doi. org/10.1186/s40104-017-0145-9 PMid:28149512

2. Mendoza-Martínez GD, Plata-Pérez FX, EspinosaCervantes R, Lara-Bueno A. Manejo nutricional para mejorar la eficiencia de utilización de la energía en bovinos. Univ Cienc. 2008; 24(1):75-87.

3. Crossland WL, Tedeschi LO, Callaway TR, Miller MD, Smith W. Effects of rotating antibiotic and ionophore feed additives on enteric methane and rumen microbial populations of steers consuming a high forage diet. J Anim Sci. 2016; 94(5):666-667. https://doi.org/10.2527/jam2016-1379

4. Ferraro SM, Mendoza GD, Miranda LA, Gutiérrez CG. In vitro gas production and ruminal fermentation of glycerol, propylene glycol and molasses. Anim Feed Sci Technol. 2009; 154(1-2):112-118. https://doi. org/10.1016/j.anifeedsci.2009.07.009
5. Liu Q, Wang C, Yang WZ, Guo G, Yang XM, He DC, et al. Effects of calcium propionate supplementation on lactation performance, energy balance and blood metabolites in early lactation dairy cows. J Anim Physiol Anim Nutr. 2010; 94(5):605-614. https:// doi.org/10.1111/j.1439-0396.2009.00945.x PMid: 19906132

6. Avila JS, Chaves AV, Hernandez-Calva M, Beauchemin KA, McGinn SM, Wang $Y$, et al. Effects of replacing barley grain in feedlot diets with increasing levels of glycerol on in vitro fermentation and methane production. Anim Feed Sci Technol. 2011; 166-167:265-268. https://doi. org/10.1016/j.anifeedsci.2011.04.016

7. Lee-Rangel HA, Mendoza GD, González SS. Effect of calcium propionate and sorghum level on lamb performance. Animal Feed Sci Technol 2012; 177(3-4):237-241. https://doi.org/10.1016/j. anifeedsci.2012.08.012 
8. Osorio-Teran AI, Mendoza-Martínez GD, MirandaRomero LA, Martínez-Gomez D, HernándezGarcía PA, Martínez-García JA. Effect of calcium propionate and monensin on in vitro digestibility and gas production. Rev Bras Zootec. 2017; 46(4):348-353. https://doi.org/10.1590/s180692902017000400011

9. Sanz-Sáez Á, Erice G, Aguirreolea J, Munoz F, Sánchez-Díaz M, Irigoyen JJ. Alfalfa forage digestibility, quality and yield under future climate change scenarios vary with Sinorhizobium meliloti strain. J Plant Physiol. 2012; 169(8):782-788. https://doi.org/10.1016/j.jplph.2012.01.010 PMid:22369772

10. Mendoza GD, Plata FX, Vázquez G, SánchezTrocino M, Hernández PA, Martínez JA. Intake and digestibility with nutritional blocks for brocked deers (Mazama americana and Mazama temama). Int J Appl Res Vet M. 2017; 15(1):26-30.

11. Geleta T, Negesse T, Abebe G, Goetsch AL. Effect of supplementing grazing Arsi-Bale sheep with molasses-urea feed block on weight gain and economic return under farmers management condition. J Cell Anim Biol. 2013; 7(10): 125-131. https://doi.org/10.5897/JCAB11.030

12. National Research Council - NRC. Nutrient Requirements of Small Ruminants: Sheep, Goats, Cervids, and New World Camelids. 1a. ed. Washington, D.C: National Academic Science; 2007.

13. Association of Official Analytical Chemist - AOAC. Methods of Analysis. 18 ed. Washington D.C.: AOAC Int.; 2011.

14. Van Soest PJ, Robertson JB, Lewis BA. Methods for dietary fibre, neutral detergent fibre, and non-starch polysaccharides in relation to animal nutrition. J Dairy Sci. 1991; 74:3583-3597. https:// doi.org/10.3168/jds.S0022-0302(91)78551-2

15. Keulen V, Young BA. Evaluation of acid-insoluble ash as a natural marker in ruminant digestibility studies. J Anim Sci. 1977; 44(2):282-287. https:// doi.org/10.2527/jas1977.442282x

16. Ellis JL, Bannink A, France J, Kebreab E, Dijkstra J. Evaluation of enteric methane prediction equations for dairy cows used in whole farm models. Glob Change Biol. 2010: 16(12):3246-3256. https:// doi.org/10.1111/j.1365-2486.2010.02188.x

17. Cambra-López M, García-Rebollar P, Estellés F, Torres A. Estimación de las emisiones de los rumiantes en Espa-a: El Factor de Conversión de Metano. Arch Zootec. 2008; 57:89-101.

18. Haque MN, Cornou C, Madsen J. Estimation of methane emission using the $\mathrm{CO} 2$ method from dairy cows fed concentrate with different carbohydrate compositions in automatic milking system. Livest Sci. 2014; 164: 57-66. https://doi.org/10.1016/j. livsci.2014.03.004
19. Brice-o PEG, Ruiz GA, Chay CAJ, Ayala BAJ, Aguilar PCF, Solorio SFJ, et al. Voluntary intake, apparent digestibility and prediction of methane production by rumen stoichiometry in sheep fed pods of tropical legumes. Anim Feed Sci Technol. 2012; 176(1-4):117-122. https://doi.org/10.1016/j. anifeedsci.2012.07.014

20. Wolin JM. A theoretical rumen fermentation balance. J Dairy Sci. 1960; 43(10):1452-1459. https://doi. org/10.3168/jds.S0022-0302(60)90348-9

21. Menke $\mathrm{KH}$, Steingass $\mathrm{H}$. Estimation of the energetic feed value obtained from chemical analysis and in vitro gas production using rumen fluid. Anim Res Dev J. 1988; 28:7-55.

22. Getachew G, Makkar HPS, Becker K. Tropical browses: contents of phenolic compounds, in vitro gas production and stoichiometric relationship between short chain fatty acid and in vitro gas production. J Agric Sci. 2002; 139(3):341-352. https://doi.org/10.1017/S0021859602002393

23. Blümmel $M$, Steinga $\beta \mathrm{H}$, Becker $\mathrm{K}$. The relationship between in vitro gas production, in vitro microbial biomass yield and $15 \mathrm{~N}$ incorporation and its implications for the prediction of voluntary feed intake of roughages. Br J Nutr. 1997; 77(6):911921. https://doi.org/10.1079/BJN19970089

24. Sall J, Lehman A, Stephens M, Creighton L. JMP start statistics: a guide to statistics and data analysis using JMP. 5th ed. SAS Institute Inc.: Cary, NC; 2012.

25. Faftine OLJ, Zanetti AM. Effect of multinutrient block on feed digestibility and performance of goats fed maize stover during the dry season in south of Mozambique. Livest Res Rural Dev. 2010; 22(9).

26. Wadhwa M, Bakshi MPS. Nutritional evaluation of urea molasses multi-nutrient blocks containing agro-industrial wastes in buffaloes. Indian J Anim Sci. $2014 ; 84(5): 544-548$.

27. Berthelot $\mathrm{V}$, Bas $\mathrm{P}$, Schmidely $\mathrm{P}$, Duvaux-Ponter $\mathrm{C}$. Effect of dietary propionate on intake patterns and fatty acid composition of adipose tissues in lambs. Small Rumin Res. 2001; 40(1):29-39. https://doi. org/10.1016/S0921-4488(00)00217-0

28. Elghandour MM, Salem AZ, Khusro A, CiprianoSalazar M, Olivares-Pérez J, Barros-Rodriguez MA, et al. Assessment of some browse tree leaves on gas production and sustainable mitigation of $\mathrm{CH} 4$ and $\mathrm{CO} 2$ emissions in dairy calves at different age. J Clean Prod. 2017; 162:1192-1199. https://doi. org/10.1016/j.jclepro.2017.06.029

29. Miranda LA, Lee-Rangel HA, Mendoza-Martínez GD, Crosby-Galván MM, Relling AE, Pinos-Rodríguez JM, et al. Influence of calcium propionate on in vitro fermentation of sorghum-based diets. Rev Fac Cien Agrar. 2017; 49(1):185-192.

30. Yao $Q$, Li $Y$, Meng $Q$, Zhou Z. The effect of calcium propionate on the ruminal bacterial comumunity composition in finishing bulls. Asian- Australasian J Anim Sci. 2017; 30(4):495-504. https://doi. org/10.5713/ajas.16.0469 PMCid:PMC5394835 